\title{
Study of Power Consumption in a Cooperative Wireless Network
}

\author{
Vahid Emamian, IEEE Senior Member
}

\begin{abstract}
A cooperative wireless network is defined to be a network where nodes cooperate in routing and/or improving the quality of transmission of each other's packets [1]-[5]. The cooperation is especially useful when the channel between a pair of nodes (source and destination) is in a deep shadow-fading state. In this situation increasing the power level may either not resolve the problem or be too power consuming, while generating interference for other receivers on the same channel. A cooperating node, which has good propagation channels to both the source and the destination, may be used to relay the packets between them. This paper presents the comparison of the average amounts of power consumed by nodes in a standard wireless network that uses single-hop transmission and a cooperative wireless network that uses two-hop transmission. It is shown that under certain conditions the ratio of the average power consumptions in the two networks, when $N$ cooperating nodes on average are available for each node, can be approximated by $k \ln N+q$. The constants $k$ and $\boldsymbol{q}$ are related to the propagation channel. For a Nakagami fading channel with parameter $\boldsymbol{m}, k=1 / \ln m$ and $\boldsymbol{q}=\mathbf{1}$, while for a shadowing channel with standard deviation $\sigma_{d B}, k=\sigma_{d B} / \sqrt{\pi}$ and $q=0.23 \sigma_{d B} \mathbf{d B}$.
\end{abstract}

Index terms: Wireless Networks, Power Analysis, Power Control, Ad-hoc Network

\section{INTRODUCTION}

A wireless network consists of a group of nodes that communicate with each other over a wireless medium of propagation. When the channel between two nodes (source: SRC and destination: DST) of such a network is in a deep shadow-fading state (see Fig 1), increasing the transmit power by the SRC can be too power consuming and result in interference for other co-channel receivers [6]. In this situation a cooperating node $(\mathrm{CN})$ may be used to relay the packets between the SRC and the DST [1]-[5]. We consider a protocol in which the cooperating node is selected from a number of nearby nodes to the SRC, such that it has the best propagation channel to the DST. The goal of this paper is to show that although an extra node becomes involved in transmitting the packets, the total average power consumed in the cooperative network is lower than that of a standard wireless network.

Manuscript received July 18, 2005; revised Feburary 24, 2006 and March 3, 2006.

V. Emamian is with Engineering Department of St Mary's University, Texas (e-mail: vemamian@stmarytx.edu).
We consider two models for the channels in our analysis; i) Nakagami fading channels, ii) shadowing channels. The problem setup is depicted in Fig 1. Referring to this figure, let $P_{S R C}^{0}$ be the power of the SRC in the direct channel (singlehop) of a standard wireless network, and $P_{C N}$ and $P_{S R C}$ be the powers, respectively, of the $\mathrm{CN}$ and the SRC in the cooperative network, for the two systems to yield the same received power at the DST. We will show that $P_{S R C}+P_{C N}<P_{S R C}^{0}$ on average.

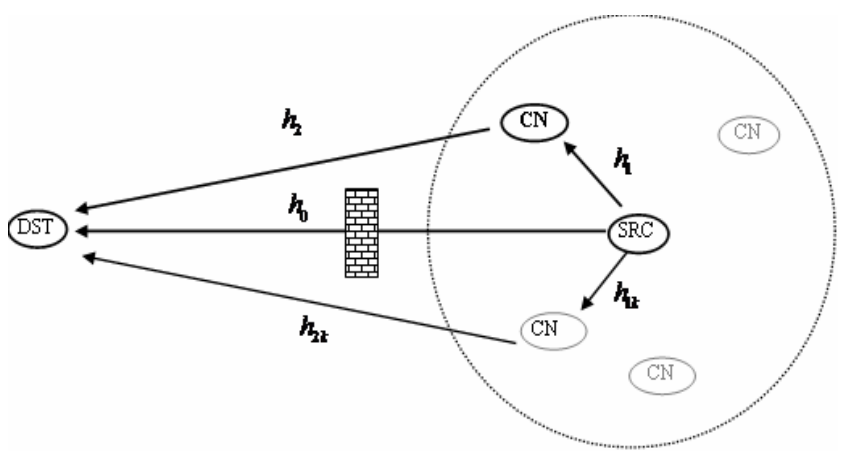

Fig 1. The relative position of the source (SRC), the destination (DST) and the cooperating nodes (CNs) in a wireless network.

\section{COMPARING POWER CONSUMPTIONS}

In this section we derive a formula for the ratio of the average power of the SRC node in the standard wireless network over the average overall power of the SRC node and the cooperating node in the relay channel to yeald the same received power level $\left(P_{R}\right)$ at the DST node. We assume that $N$ cooperating nodes are available for each SRC node, on the average. In Section II. $A$ we assume that all nodes face independent Nakagami fast fading channels, while in Section II. $B$ shadowing channels will be considered. Because of the intractability of the analysis, the case of composite shadowfading channels is not considered here.

\section{A. Nakagami fast-fading channels}

Let $h_{0}, h_{1 k}$, and $h_{2 k}$ be the channels between the SRC and the DST, the SRC and the $k^{\text {th }} \mathrm{CN}$, and the DST and the $k^{\text {th }} \mathrm{CN}$ respectively (see Fig 1), and $\Omega_{0}, \Omega_{1 k}$ and $\Omega_{2 k}$ be the corresponding area mean powers [6]. For simplicity we assume equal area mean powers associated with the radius of 
the neigborhood, i.e. $\Omega_{2 k} \cong \Omega_{2}, \quad k=1, \ldots, N$. The magnitudes of $h_{0}, h_{1 k}$ and $h_{2 k}$ have Nakagami distribution, i.e. ${ }^{1}$

$$
\begin{array}{r}
f_{\left|h_{0}\right|}(r)=\frac{2}{\Gamma(m)}\left(\frac{m}{\Omega_{0}}\right)^{m} r^{2 m-1} e^{-m r^{2} / \Omega_{0}} \\
f_{\left|h_{j k}\right|}(r)=\frac{2}{\Gamma(m)}\left(\frac{m}{\Omega_{j k}}\right)^{m} r^{2 m-1} e^{-m r^{2} / \Omega_{j k}}, j=1,2, r>0
\end{array}
$$

For a fair comparison, we set the same received power at the DST in both cooperative and standard networks. Let $P_{R}$ be the received power at the $\mathrm{CN}$ and the DST in both networks, then we have

$$
P_{S R C}^{0} \propto \frac{P_{R}}{\left|h_{0}\right|^{2}}, P_{C N} \propto \frac{P_{R}}{\left|h_{2}\right|^{2}} \text {, and } P_{S R C} \propto \frac{P_{R}}{\left|h_{1}\right|^{2}}
$$

We define the power consumption ratio of the two networks to be

$$
\alpha \triangleq \frac{E\left[P_{S R C}^{0}\right]}{E\left[P_{S R C}\right]+E\left[P_{C N}\right]},
$$

where $E[$.$] is the expectation operator.$

We also define $S_{0} \triangleq \frac{1}{\left|h_{0}\right|^{2}}$ and $S_{j k} \triangleq \frac{1}{\left|h_{j k}\right|^{2}}, j=1,2$. The probability density function (PDF) of $S_{0}$ is then given by

$$
f_{S_{0}}(s)=\frac{1}{s \Gamma(m)}\left(\frac{m}{\Omega_{0} s}\right)^{m} e^{-m / \Omega_{0} s}, s>0
$$

and from this the expected values of $S_{0}$ is given by

$$
E\left[S_{0}\right]=\frac{m}{\Omega_{0}(m-1)} .
$$

Similarly

$$
E\left[S_{1 k}\right]=\frac{m}{\Omega_{1 k}(m-1)} .
$$

Since the CN has the best channel to the DST node, we have $\left|h_{2}\right|=\max _{k}\left\{\left|h_{2 k}\right|\right\}, k=1,2, \ldots, N$. The cumulative density function (CDF) of $\left|h_{2}\right|$ is then given by ${ }^{2}$

$$
F_{\left|h_{2}\right|}(s \mid N)=\operatorname{Pr}\left(\left|h_{2}\right|<s\right)=\prod_{k=1}^{N} \operatorname{Pr}\left(\left|h_{2 k}\right|<s\right)=\left[F_{\left|h_{2 k}\right|}(s)\right]^{N} \text {. }
$$

On the other hand, since $\left|h_{2 k}\right|, k=1,2, \ldots, N$ have Nakagami distributions, using (1) it can be shown that the CDF of $\left|h_{2 k}\right|$ is given by

$$
F_{\left|h_{2 k}\right|}(s)=1-\frac{1}{\Gamma(m)} \Gamma\left(m, \frac{m s}{\Omega_{2 k}}\right) .
$$

Using (6) and assuming that $\left|h_{2 k}\right|, k=1,2, \ldots, N$ are i.i.d., the CDF of $\left|h_{2}\right|$ is given by

\footnotetext{
${ }^{1}$ Gamma function is defined as $\Gamma(m, t) \triangleq \int_{t}^{\infty} e^{-\lambda} \lambda^{m-1} d \lambda$ and
} $\Gamma(m) \triangleq \Gamma(m, 0)$.

${ }^{2}$ Mathematica is used for simplification of all formulas. Matlab is also used as the main computational tool.

$$
F_{\left|h_{2}\right|}(s)=\left(1-\frac{1}{\Gamma(m)} \Gamma\left(m, \frac{m s}{\Omega_{2}}\right)\right)^{N},
$$

Defining $S_{2}=\frac{1}{\left|h_{2}\right|}$ and by using (7) it can be shown that the PDF of $S_{2}$ is expressed as

$$
f_{S_{2}}(s)=\frac{N}{s \Gamma(m)}\left(\frac{m}{\Omega_{2} s}\right)^{m} e^{-m / \Omega_{2} s}\left(1-\frac{1}{\Gamma(m)} \Gamma\left(m, \frac{m}{\Omega_{2} s}\right)\right)^{N-1}
$$

From (8) the expected value of $S_{2}$ is given by

$$
E\left[S_{2}\right]=\frac{m N}{\Omega_{2} \Gamma(m)} \int_{0}^{\infty} t^{m-2} e^{-t}\left(1-\frac{1}{\Gamma(m)} \Gamma(m, t)\right)^{N-1} d t .
$$

Now, the power consumption ratio of the two networks, which was given in (2), is given by

$$
\alpha=\frac{E\left[S_{0}\right]}{E\left[S_{1}\right]+E\left[S_{2}\right]} .
$$

Substituting (4), (5) and (9) in (10), the inverse of power consumption ratio of the two channels is given by ${ }^{3}$

$$
\alpha^{-1}=\frac{\Omega_{0}}{\Omega_{1}}+\frac{\Omega_{0}}{\Omega_{2}} \frac{N(m-1)}{\Gamma(m-1)} \int_{0}^{\infty} t^{m-2} e^{-t}\left(1-\frac{1}{\Gamma(m)} \Gamma(m, t)\right)^{N-1} d t(11)
$$

Assuming $\Omega_{0} \cong \Omega_{2}{ }^{4}$, and using the fact that $\Omega_{0} \ll \Omega_{1}{ }^{5}$, Eq. (11) can be simplified to

$$
\alpha^{-1} \cong \frac{N(m-1)}{\Gamma(m)} \int_{0}^{\infty} t^{m-2} e^{-t}\left(1-\frac{1}{\Gamma(m)} \Gamma(m, t)\right)^{N-1} d t .
$$

By computing the first few terms of the Taylor series of $\alpha$ from Eq. (12) around $N=1$, it can be shown that for $1<m<3$, $\alpha$ can be well approximated by

$$
\alpha \cong k \ln N+q,
$$

where $k=1 / \ln m$ and $q=1$. The average number of the cooperating nodes in the network, $N$, is a function of the total number of nodes. For example, let $N_{u}$ nodes be uniformly distributed in a disk with radius $R$ and let $r$ be the cooperation distance ${ }^{6}$, then $N \cong N_{u} r^{2} / R^{2}$ if $r \ll R$.

\footnotetext{
${ }^{3}$ We have also assumed that $\Omega_{1 k} \cong \Omega_{1}$ for the $\mathrm{CN}$. Since $\Omega_{1 k} \gg \Omega_{0}$ for all values of $k$, and we eventually ignore the term $\Omega_{0} / \Omega_{1}$, this assumption does not make any difference in the final results.

${ }^{4}$ Since the distance between a CN and the DST is almost equal to the distance between the SRC and the DST, this assumption is reasonable.

${ }^{5} \Omega_{1} \propto r^{-\beta}$ and $\Omega_{0} \propto D^{-\beta}$ where $\beta$ is the path-loss exponent and $r$ is the distance between SRC and $\mathrm{CN}$ and $D$ is the distance between DST and SRC, hence $\Omega_{0} / \Omega_{1}=(r / D)^{\beta}$. Since $r \ll D$ and $\beta>2$, we have $\Omega_{0} \ll \Omega_{1}$.

${ }^{6} \mathrm{We}$ assume that two nodes can cooperate if the distance between them is less than a cooperation distance $(r)$.
} 
Fig 2 shows the value of $\alpha$ versus the number of cooperating nodes $(N)$ for $m=1.5$ (from Eqs. (12) and (13)). For a network of radius $5 \mathrm{~km}$, wherein 1000 nodes are uniformaly distributed over the area of the disk, the average number of the cooperating nodes is about 1.6 , when the cooperation distance is $200 \mathrm{~m}$. The figure shows that for $N=1.6$, the average power consumption in the cooperative network is almost half the average power consumtions in the standard network.

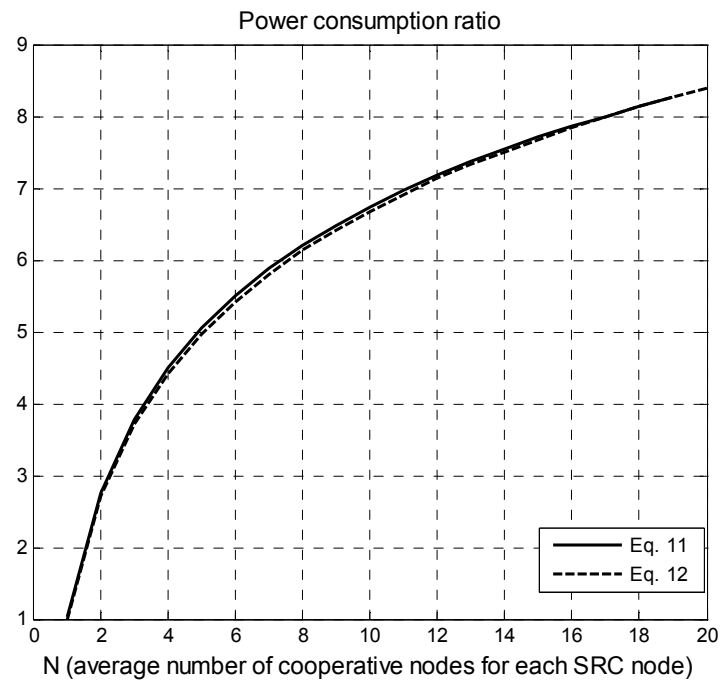

Fig 2. $\alpha$ : Ratio of the average power consumption of the standard wireless network over that for the cooperative network versus average number of the cooperating nodes around each SRC node.

\section{B. Shadowing channels}

In this section we derive an expression for the power consumption ratio in the presence of lognormal shadowing and no fading. For the shadowing channel, the area mean powers $\Omega_{0}, \Omega_{1 k}$ and $\Omega_{2 k}$ are lognormal random variables, i.e.

$\ln \Omega_{0} \sim N\left(\mu_{0}, \sigma\right), \ln \Omega_{1 k} \sim N\left(\mu_{1 k}, \sigma\right), \ln \Omega_{2 k} \sim N\left(\mu_{2 k}, \sigma\right)$, where $N(\mu, \sigma)$ is a normal distribution with mean $\mu$ and standard deviation $\sigma$. We assume that all channels have the same shadowing standard deviation, and all cooperating nodes have almost the same average area mean power from the DST node $\left(\mu_{21} \approx \mu_{22} \approx \ldots \approx \mu_{2 N} \triangleq \mu_{2}\right)$. Let $\Omega_{1}$ and $\Omega_{2}$ be the area mean power at the $\mathrm{CN}$ from the SRC and the DST respectively. Again, in this case we assume that the transmitted power level is proportional to the received threshold power over channel's power, i.e.

$$
P_{S R C}^{0} \propto \frac{P_{t h}^{R}}{\Omega_{0}}, P_{C N} \propto \frac{P_{t h}^{R}}{\Omega_{2}}, P_{S R C} \propto \frac{P_{t h}^{R}}{\Omega_{1}} .
$$

Defining $\alpha_{d B} \triangleq 10 \log \left[P_{S R C}^{0} /\left(P_{C N}+P_{S R C}\right)\right]$, it can be written as $^{7}$

\footnotetext{
${ }^{7}$ For small values of $x$ we have $\ln (1+\mathrm{x}) \approx \mathrm{x}$.

Since $\Omega_{2} / \Omega_{1} \ll 1$, we simply ignored it. This was discussed in the previous section for $\Omega_{0} / \Omega_{1}$.
}

$$
\begin{aligned}
& \alpha_{d B} \cong \frac{10}{\ln 10} \ln \frac{P_{t h}^{R} / \Omega_{0}}{P_{t h}^{R} / \Omega_{2}+P_{t h}^{R} / \Omega_{1}} \cong \\
& \frac{1}{\xi}\left(\ln \Omega_{2}-\ln \Omega_{0}-\frac{\Omega_{2}}{\Omega_{1}}\right) \cong \frac{1}{\xi}\left(\ln \Omega_{2}-\ln \Omega_{0}\right)
\end{aligned}
$$

where $\xi \triangleq \ln 10 / 10$.

Now we need to find the distribution of $\ln \Omega_{2}$. Since $\ln \Omega_{2}=\max _{k}\left\{\ln \Omega_{2 k}\right\}, k=1,2, \ldots, N$, assuming that $\ln \Omega_{2 k}$ are normal i.i.d with mean $\mu_{2}$ and standard deviation $\sigma$, the CDF of $\ln \Omega_{2}$ is given by

$$
\begin{aligned}
& F_{\ln \Omega_{2}}(x)=\operatorname{Pr}\left(\ln \Omega_{2}<x\right)=\prod_{k=1}^{N} \operatorname{Pr}\left(\Omega_{2 k}<x\right)= \\
& {\left[F_{\ln \Omega_{2 k}}(x)\right]^{N}=\frac{1}{2^{N}}\left[1+\operatorname{erf}\left(\frac{\left(x-\mu_{2}\right)}{\sqrt{2} \sigma}\right)\right]^{N},}
\end{aligned}
$$

where $\operatorname{erf}(t) \triangleq \frac{2}{\sqrt{\pi}} \int_{0}^{t} e^{-z^{2}} d z$. The PDF of $\ln \Omega_{2}$ is then given by

$$
\begin{aligned}
& f_{\ln \Omega_{2}}(x)=d F_{\ln \Omega_{2}}(x) / d x= \\
& \frac{1}{\sqrt{2 \pi} \sigma} \frac{N}{2^{N-1}} e^{-\left(x-\mu_{2}\right)^{2} / 2 \sigma^{2}}\left[1+\operatorname{erf}\left(\frac{\left(x-\mu_{2}\right)}{\sqrt{2} \sigma}\right)\right]^{N-1} .
\end{aligned}
$$

Using (15), the expected value of $\ln \Omega_{2}$ is obtained from

$$
E\left[\ln \Omega_{2}\right]=\mu_{2}+\sigma G(N),
$$

where $G(N)$ is defined to be

$$
G(N) \triangleq \frac{N}{2^{N-2} \sqrt{2 \pi}} \int_{-\infty}^{+\infty} t e^{-t^{2}}(1+\operatorname{erf}(t))^{N-1} d t .
$$

Defining $\bar{\alpha}_{d B} \triangleq E\left[\alpha_{d B}\right]$ and using (14), we have

$$
\bar{\alpha}_{d B}=\frac{1}{\xi}\left(E\left[\ln \Omega_{2}\right]-E\left[\ln \Omega_{0}\right]\right) .
$$

Knowing that $E\left[\ln \Omega_{0}\right]=\mu_{0}$ and substituting (16) in (18) we get

$$
\bar{\alpha}_{d B}=\frac{\mu_{2}-\mu_{0}}{\xi}+\frac{\sigma}{\xi} G(N)
$$

For simplicity we assume that the value of $\mu_{2}$ (average area mean power at the $\mathrm{CN}$ ) is almost equal to $\mu_{0}$ (average area mean power at the SRC), because they are approximately located at the same distance from the $\mathrm{DST}^{8}$. Therefore

$$
\bar{\alpha}_{d B} \cong \frac{\sigma}{\xi} G(N) \text {. }
$$

It can be shown, by model fitting, that for $1<N<30, G(N)$ can be well approximated by $(\ln N+0.4) / \sqrt{\pi}$. Therefore

$$
\bar{\alpha}_{d B} \cong k \ln N+q \text {. }
$$

where $k=\sigma_{d B} / \sqrt{\pi}, q=0.23 \sigma_{d B}$ and $\sigma_{d B}=\sigma / \xi$.

\footnotetext{
${ }^{8}$ At least $\mu_{2}$ is not less than $\mu_{0}$ because the channel from the best cooperating node $(\mathrm{CN})$ to the DST would always have a larger average area mean power than the channel from the SRC to the DST. When $\mu_{2}>\mu_{0}$ the performance is even better.
} 
For $N=1,2,3 ; G(N)$ has closed-form values that are given in TABLE . The value of $\bar{\alpha}_{d B}$ versus the number of cooperating nodes (from Eqs. (20) and (21)) is shown in Fig 3, for a shadowing standard deviation of $8 \mathrm{~dB}\left(\sigma_{d B}=\sigma / \xi=8 \mathrm{~dB}\right)$. From this figure we notice that the relative performance of the cooperative network over the standard network improves in the presence of shadowing.

TABLE I.

The Value OF G(N) For N=0, 1, 2, 3.

\begin{tabular}{|c|c|c|c|}
\hline$N$ & 1 & 2 & 3 \\
\hline$G(N)$ & 0 & $1 / \sqrt{\pi}$ & $3 / 2 \sqrt{\pi}$ \\
\hline
\end{tabular}

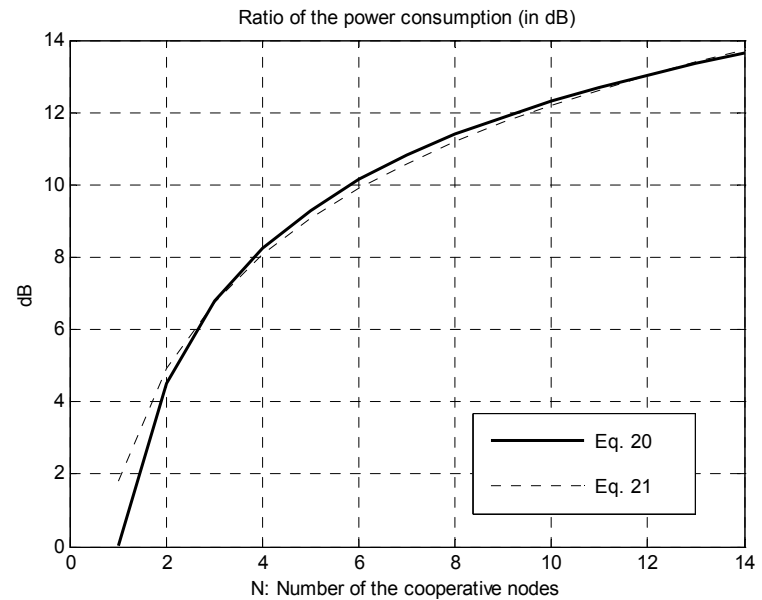

Fig 3. $\bar{\alpha}_{d B}$ : Ratio of the power consumption (in $\mathrm{dB}$ ) in the standard network over that for the cooperative network versus average number of the cooperating nodes.

\section{CONCLUDING REMARKS}

This article presented the comparison of the average amount of power consumed by nodes in a standard and a cooperative wireless network, in which a single best node is used to relay the signal between a source and destination.
It was shown that the ratio of the average power consumption in the two networks, when $N$ cooperating nodes on average are available for each node, is approximately $k \ln N+q$, for Nakagami and lognormal shadowing channels. The constants $k$ and $q$ are related to the type of the channels.

\section{REFERENCES}

[1] [1] P. Gupta, and P. R. Kumar, "Critical power for asymptotic connectivity," Proceedings of the 37th IEEE Conference on Decision and Control, 1998, vol. 1, pp 1106-1110.

[2] [2] V. Emamian, and M. Kaveh, "Combating shadowing effects for systems with transmitter diversity by using collaboration among mobile users", Proceedings of the International Symposium on Communications, no. 9.4, pp. $105.1 \sim 105.4$, Nov. 13-16, 2001, Taiwan.

[3] [3] J.N. Laneman, and G.W. Wornell, "Energy-efficient antenna sharing and relaying for wireless networks," IEEE Wireless Communications and Networking Conference, vol. 1, 2000, pp. 7-12.

[4] [4] V. Emamian, and M. Kaveh, "Comparing Power Consumption of Collaborative and non-Collaborative Systems", Proceedings of the 36th Asilomar Conference on Signals, Systems and Computers, Nov 3-6, 2002.

[5] [5] A. Sendonaris, E. Erkip, and B. Aazhang, "Increasing uplink capacity via user cooperation diversity," Proceedings of IEEE International Symposium on Information Theory, 1998, pp 156.

[6] [6] G. L. Stuber, Principles of Mobile Communication. Boston, MA: Kluwer, 1996.

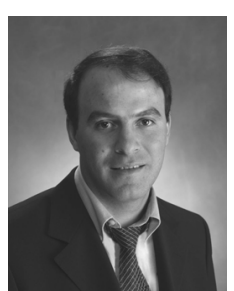

Vahid Emamian received his B.S. and M.S. degrees in electrical engineering from Sharif University of Technology, Tehran, in 1996 and 1998, respectively, and his Ph.D. degree from the Department of Electrical and Computer Eng. at the University of Minnesota in 2003. His research interests include wireless communications, cooperative wireless networks, and digital signal processing. He is currently an assistant professor of electrical engineering at St Mary's University in Texas. Prior to joining St Mary's in 2003, Dr. Emamian has been a research assistant at the Department of Electrical and Computer Engineering, University of Minnesota, from 1999 to 2003, where he worked on cooperative wireless networks. He has also received MSEIP award from the Education Department in 2005. 